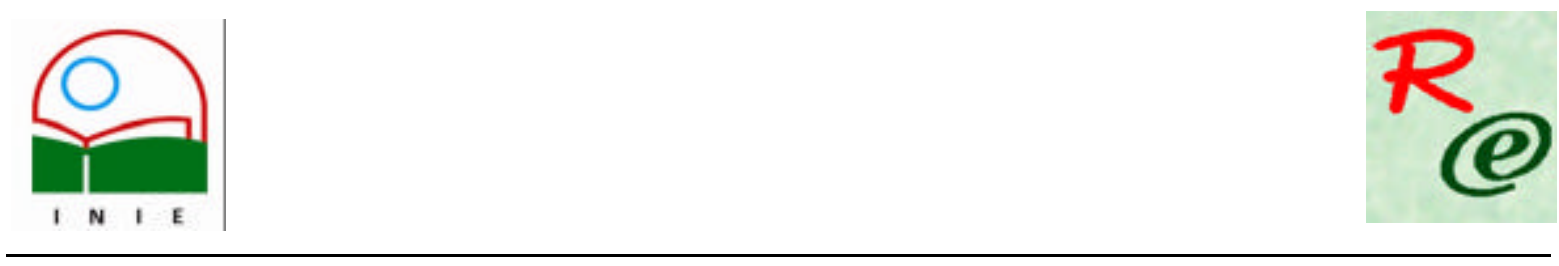

Actualidades Investigativas en Educación

Revista Electrónica publicada por el

Instituto de Investigación en Educación

Universidad de Costa Rica

ISSN 1409-4703

http://revista.inie.ucr.ac.cr

COSTA RICA

\title{
LAS ESTRATEGIAS DE APRENDIZAJE. RADIOGRAFÍAS NECESARIAS PARA SU COMPRENSIÓN.
}

THE STRATEGIES OF LEARNING. X-RAY NECESSARY FOR THEIR UNDERSTANDING.

Volumen 9, Número 2

pp. $1-26$

Este número se publicó el 30 de agosto 2009

Armenio Pérez Martínez

La revista está indexada en los directorios:

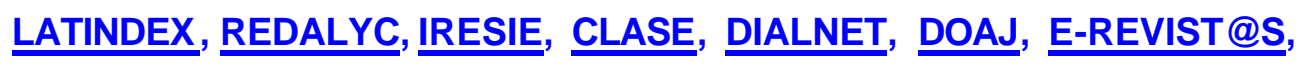

La revista está incluida en los sitios:

REDIE, RINACE, OEI, MAESTROTECA, PREAL, HUASCARAN, CLASCO 


\title{
LAS ESTRATEGIAS DE APRENDIZAJE. RADIOGRAFÍAS NECESARIAS PARA SU COMPRENSIÓN
}

THE STRATEGIES OF LEARNING. X-RAY NECESSARY FOR THEIR UNDERSTANDING

\author{
Armenio Pérez Martínez ${ }^{1}$ \\ "el estudiante al egresar de las aulas universitarias \\ debe ser capaz de movilizar sus recursos personales, \\ adecuándolos al contexto laboral específico, \\ mediante el uso de herramientas intelectuales y sociales, \\ así como debe ser capaz de autogestionarse nuevos aprendizajes \\ que le permitan responder acertadamente \\ a las demandas crecientes de la práctica".
}

UNESCO. 1998

\begin{abstract}
Resumen: En la actualidad resulta muy frecuente referirse a las estrategias de aprendizaje desde distintos enfoques epistemológicos, sin que exista unanimidad en cuanto a los criterios que sirven para caracterizar este concepto. Este ensayo es una oportunidad de acercarnos a esta realidad, mostrando algunas de sus aristas. Se realiza un acercamiento a los antecedentes históricos y conceptuales, así como se muestran las consideraciones propias. Como colofón se plasma la propuesta de una nueva forma de concebir las estrategias de aprendizaje, donde se trascienda los postulados constructivistas y se conciba el desarrollo integral de las mismas desde el enfoque histórico-cultural.
\end{abstract}

Palabras Claves: ESTRATEGIAS DE APRENDIZAJE, CONSTRUCTIVISMO, ENFOQUE HISTÓRICO CULTURAL.

\begin{abstract}
At the present time it is very frequent to listen to speak of learning strategies from different you focus epistemology, without unanimity exists as for the approaches that are good to characterize this concept. It is this rehearsal an opportunity to come closer to this reality, showing some of their edges. He/she is carried out an approach to the historical and conceptual antecedents, as well as this author's considerations are shown. As colophon the proposal in a new way is captured of conceiving the learning strategies, where you transcends the postulates constructivist and the integral development of the same ones is conceived from the historical-cultural focus.
\end{abstract}

Key words: LEARNING STRATEGIES, CONSTRUCTIVE, HISTORICAL - CULTURAL FOCUS.

\footnotetext{
Labora en el Centro de Estudios Educacionales. Universidad de Ciego de Ávila. Cuba.
}

Dirección electrónica: armenito@economia.unica.cu

Artículo recibido: 24 de febrero, 2009

Aprobado: 24 de agosto, 2009 


\section{Introducción}

Las trasformaciones en el panorama social, económico, político y técnico-organizativo del mundo contemporáneo provocan repercusiones en el ámbito educativo que trata de ajustarse a estas necesidades cambiantes. Desde esta perspectiva se clarifica el carácter insoslayable ce la individualización de la educación, al comprender, el proceso educativo como un proceso formativo que del modo más sistemático se dirige a la formación social de las nuevas generaciones y en él, la persona como estudiante se instruye, desarrolla y educa. Si se valoran los retos actuales a los sistemas educativos en cada nación, se aprecia que estos no operan solo dentro del ámbito profesional, sino que trascienden a complejos constructos como la ideología y a formaciones psíquicas superiores como la autoeducación y el autodesarrollo. Las personas egresadas demandan cada día profesionales más competentes, adaptables al cambio, con creatividad y con capacidad de transformar. Se afirma que nuestras universidades deben de formar una persona más capaz, autónoma y con crecimiento personológico. Esta idea resalta la necesidad de formar en las aulas un sujeto activo, con un desarrollo integral que puede ser puesto de manifiesto en todas sus esferas de actuación dentro de la vida cotidiana, garantizando su éxito profesional, familiar, intelectual y social.

La educación es el proceso que pretende preparar a los sujetos para su futuro desempeño profesional y social, por tanto, es permanente en la vida de cada persona. Tradicionalmente, el proceso de enseñanza-aprendizaje ha sido utilizado como la vía idónea para trasmitir información y ser interiorizada por parte del estudiante, asumiendo este una posición pasiva en el proceso, y a grupo docente se le han asignado diversos roles: el de trasmisor de conocimientos, el de animador, el de supervisor, o guía del proceso de aprendizaje e incluso el de investigador educativo. Hoy día es un clamor social que la tarea docente no debe restringirse a una mera transmisión de información.

Dentro de la investigación psicológica en la actualidad se ha posibilitado el arraigo de las ideas constructivistas y las estrategias de aprendizaje como elemento determinante en el proceso de enseñanza-aprendizaje. El constructivismo ha brindado la posibilidad de valorar al estudiante como el ser activo que construye el conocimiento, centrado en su actividad mental y conocimiento previo, donde la función docente es engarzar los procesos de 
construcción del conocimiento de la población estudiantil con el saber colectivo culturalmente organizado.

Las estrategias de aprendizaje apreciadas como acciones conscientes, cuyo propósito es el aprendizaje y la resolución de problemas académicos, han servido para que las estudiantes y los estudiantes enfrenten con mayor cantidad de recursos intelectuales las situaciones docentes en el aula. A pesar de ello el constructivismo y las estrategias de aprendizaje plantean una limitación del desarrollo de esta población al plano intelectual y al dominio de esquemas y algoritmos de solución.

En este ensayo se denomina radiografías a la posibilidad de analizar esta realidad interior de las estrategias de aprendizaje, en estrecha relación con otros conceptos dentro del proceso de enseñanzaaprendizaje y en el contexto del desarrollo humano integral.

Es por ello que se propone realizar un análisis de las estrategias de aprendizaje, sus posibilidades, así como se pone a consideración algunas alternativas de accionar desde el contexto educativo para promover aprendizajes significativos y cooperativos.

\section{Desarrollo}

Diversos autores marxistas, entre ellos Bozhovich (1985) y Vigostky (1987) han acuñado la idea de que la educación como proceso permanente que posibilita capacitar al hombre para la vida, cumple una función esencial en el crecimiento personal del sujeto. El proceso de formación y despliegue de lo humano pone una naturaleza social, interactiva y modificable, para resaltar la importancia de la educación como proceso inherente del ser humano. En la obra de estos autores se hace referencia a la relación entre la educación y el desarrollo del sujeto, concibiendo que la educación pauta las premisas para el desarrollo personológico de las personas. La concepción vigotskiana de la relación entre el desarrollo y la educación, sustentada en una teoría que explica consecuentemente los principales postulados referidos a la esencia social de lo psíquico y a su desarrollo histórico, plantea que la educación debe estar orientada al futuro no hacia el pasado.

El desarrollo es el proceso integral y sistémico donde se reestructuran los contenidos psicológicos, dando lugar a formaciones psíquicas superiores cualitativamente nuevas, Volumen 9, Número 2, Año 2009, ISSN 1409-4703 
posibilita una mayor regulación sobre el comportamiento y prepara al sujeto para su futuro. La psicología ha centrado su análisis alrededor de este proceso, siendo Vigotsky (1989) uno de los precursores de esta idea, al considerar que los elementos psicológicos se presentan en una integración en desarrollo denominada personalidad.

En la literatura sobre la temática existen dos vías para explicar el desarrollo humano. Considerar que es necesaria la maduración de las estructuras nerviosas para desarrollar el aprendizaje, o realizar un aprendizaje estimulador del desarrollo bio-psico-social. Se puede estimular el desarrollo partiendo de la educación de los sujetos. Se muestran ejemplos en nuestra sociedad como las vías no formales donde el aprendizaje estimula el desarrollo de los menores, además si se considera que el ser humano está en constante desarrollo y aprendizaje se puede afirmar que la enseñanza y la educación son procesos que favorecen el desarrollo de los sujetos.

Los modelos educativos, en su devenir histórico, se han encontrado estrechamente relacionados a la idea de que su esencia es la selección de estudiantes más competentes. En estos momentos se afirma que la escuela debe cambiar su función hacia la formación de sujetos más capaces, motivados, competentes y desarrollados íntegramente. En esta opinión es que podemos inscribir nuestro criterio.

Se convierte en un reto para la educación institucionalizada diseñar y proponer modelos, situaciones de aprendizaje, etc., que propicien al máximo el desarrollo de las personas, que potencien sus capacidades para enfrentar la vida constructiva, crítica, reflexiva, con autonomía, autogestión, de forma tal que se creen las bases para su constante crecimiento personal y autoperfeccionamiento.

Una enseñanza adecuadamente desarrollada es un elemento esencial para la educación de la personalidad. En ella, además de obtener conocimientos, la población estudiantil podrá desarrollar intereses, aprender a expresarse, a defender sus criterios, a conformar opiniones propias, a plantearse interrogantes hacia la realidad, a educar su persistencia, su autocrítica y muchas otras particularidades esenciales para el des arrollo de la personalidad. 
Una de las tendencias que estudia el problema del desarrollo con mayor fuerza es la escuela humanista de Psicología y dentro de ella C. Rogers y G. Allport (Pérez Cabaní, 2000). Rogers enfatiza que en el sujeto existen dos tendencias fundamentales en el organismo: a la autoconservación y al desarrollo. Considera el desarrollo como esencial en seres humanos así como su capacidad de insertarse al mundo que los rodea en busca de su desarrollo, considerando que solo hay que orientar estas fuerzas que lo guían.

Para G. Allport (Pérez Cabaní, 2000) un aspecto fundamental del desarrollo es el alcance de una personalidad madura. Distingue tres criterios como esenciales para determinar el desarrollo de esa personalidad. En primer lugar, señala la gran variedad de intereses autónomos, implicando la participación "con calor" en toda empresa que ha adquirido valor para la persona. Reconoce la "auto-objetivación" como esa capacidad de comprensión de sí mismo. Concluye afirmando la importancia de una "filosofía unitaria de la vida", mostrándole su lugar entre todas las cosas.

El enfoque gestaltista, dentro de la Psicología, orienta el estudio de la personalidad como un todo. Elemento de gran interés frente a la fragmentación que reina en buena parte de la Psicología actual, resaltando que el estudio de las unidades conservan la especificidad del todo.

En la Psicología materialista dialéctica aparece con más fuerza la relación que se establece entre las condiciones internas y externas para el desarrollo y la formación de la personalidad integral. L. I. Bozhovich planteó el desarrollo como: "proceso complejo, cuya comprensión exige siempre el análisis, no solo de las condiciones objetivas que influyen sobre el niño, sino también de las particularidades ya formadas de su psiquis, a través de las cuales se refractan las influencias de estas condiciones" (Bozhovich, 1985, p. 99). L. S. Vigotsky desarrolló esta idea al exponer que "las influencias del medio, varían en dependencia de las propiedades psicológicas del niño formadas anteriormente, a través de los cuales se refractan". (Bozhovich, 1985, p. 75).

La comprensión de este proceso fuera del marco materialista y dialéctico es prácticamente imposible. Es un proceso de crecimiento y en constante cambio durante la historia vital del sujeto. En uno de sus acercamientos a este proceso L. S. Vigotsky (1989, p. 104) lo define Volumen 9, Número 2, Año 2009, ISSN 1409-4703 
como: "la totalidad de los procesos que llevan al desenvolvimiento del organismo individual en función del tiempo. Consta de dos aspectos esenciales: maduración genéticamente y aprendizaje".

Otro concepto, que implica un aporte de valor en los intentos de comprender el desarrollo humano, es el de zona de desarrollo próximo, concebida como: "distancia entre el nivel de desarrollo actual determinado por la capacidad de resolver independientemente un problema y el nivel de desarrollo potencial determinado mediante la resolución de un problema bajo la guía de un adulto o colaboración de un compañero" (Vigotsky, 1987, p. 33). Este concepto permite comprender cómo el sujeto a través de las interacciones es capaz de resolver tareas que solo no podía e interioriza las herramientas psicológicas surgidas en esa relación, que le permitirán realizar la tarea por sí solo.

Cabe destacar el principio de la doble formación de los procesos psíquicos en el que se enfatiza que todo proceso antes de convertirse e intrapsíquico es social, interpsíquico, es decir, surge de las relaciones que establece el individuo en su estado actual con el medio potenciador. Se reconoce el papel de la interiorización en este proceso de apropiación del entorno social: "Llamamos interiorización a la reconstrucción interna de una operación externa" (Vigotsky, 1968, p. 88).

Se considera otro aporte vital de este autor el concepto de Situación Social de Desarrollo, definido como: "aquella combinación especial de los procesos internos del desarrollo y las condiciones externas, que es típica en cada etapa y que condicionan la dinámica del desarrollo durante el correspondiente periodo evolutivo y nuevas formaciones psicológicas". (Bozhovich, 1985, p. 99).

Al concebir el desarrollo como un proceso ininterrumpido de cambios y progresos en las distintas operaciones y formaciones de la personalidad, implicadas en sus funciones reguladoras y autorreguladoras, se induce al abordaje del desarrollo desde una perspectiva personológica. En el proceso de educación, junto al redimensionamiento de los currículos, de los recursos pedagógicos de as profesoras y los profesores para dictar sus dases, el conocimiento de las propias operaciones que el conocimiento exige, de otros elementos del proceso mismo de la impartición del conocimiento y el aprendizaje de este por parte del Volumen 9, Número 2, Año 2009, ISSN 1409-4703 
grupo estudiantil ${ }_{2}$ es necesario ampliar las vías para el desarrollo de la personalidad escolar, estimular su iniciativa, su persistencia, su capacidad de polémica, su seguridad en sí mismo, su autonomía y gestión personalizada, entre otros que tendrán un significado esencial en la forma en que el estudiantado utilice los conocimientos.

Esta forma de abordar el desarrollo personológico es una forma de concebir de desarrollo humano: este es un proceso complejo, multideterminado, único, que cada sujeto vivencia de manera individual. Afirmar el carácter de proceso del mismo nos lleva a precisar la existencia de una temporalidad ilimitada, enmarcada solo por los propios límites de la vida del sujeto, también emerge la idea de lo individual, lo complejo, lo constructivo, garantizando que el sujeto sea una entidad sui generis, donde se combinen de manera peculiar contenidos psicológicos.

Los procesos de desarrollo de la personalidad son intrínsecos a sus formas constitutivas en cada uno de sus momentos de existencia. El desarrollo ocurre como resultado de las propias contradicciones que aparecen en la personalidad, en las cuales lo externo no representa más que un momento constitutivo de la configuración en desarrollo.

La finalidad del desarrollo es el dominio del entorno y de sí mismo, expresado en la autorregulación del comportamiento, siendo capaz de adaptarse y trascender a la inmediatez y al impulso, contando con recursos superiores de control que sirven para organizar su personalidad. A lo largo de la vida el sujeto construye significados y atribuye sentidos a los objetos y hechos que lo rodean, permitiéndole entonces actuar en consecuencia. Además la estructuración e integración de los contenidos y funciones psicológicas permiten una mejora en incremento, lo que posibilita que el sujeto pueda planificar sus acciones y su comportamiento.

La forma específicamente humana de aprendizaje es siempre un proceso interactivo, mediado por la existencia de una cultura que se va haciendo propia, la existencia de las otras personas y de uno mismo. Existe aquí una unidad dialéctica entre aprendizaje y desarrollo. El aprendiz facilita, propicia y se manifiesta como fuente de desarrollo, va adelante y abre el camino. Cada nuevo nivel de desarrollo es el resultado y punto de partida para los continuos aprendizajes que el sujeto realiza en su vida. 


\section{Radiografía Número 1. La relación Aprendizaje-Desarrollo}

¿Cómo se ha concebido tradicionalmente el aprendizaje?

Por lo general, en la literatura se recogen un gran número de trabajos donde suele entenderse como aprendizaje aquellos cambios bastante estables, expresados en la conducta o inferidos en las estructuras de conocimientos de los sujetos.

Así, para algunos autores dentro del enfoque conductista como W. Watson y B. Skinner, aprender se concibe en términos de adquisición y modificación de conductas y el énfasis descansa en el ambiente y en la organización de influencias externas. Para otros, consiste en un proceso en el que se desarrollan conocimientos, perspectivas y formas de pensar, el énfasis se encuentra en la actividad mental que organiza y construye. Estos modelos de aprendizaje - el conductismo, el cognitivismo y el racionalismo - a pesar de los resultados que han ofrecido a la Psicología Educativa muestran una visión limitada del proceso, en tanto que maximizan los elementos cognitivos, restringiendo el aprendizaje a un desarrollo del saber, a un proceso de adaptación a la vida.

Según nuestro criterio, aprender es un proceso permanente de cambios dados en el devenir histórico, en nuestra historia individual el aprendizaje puede ser concebido como un proceso activo, personal, de construcción y reconstrucción de conocimientos, de descubrimientos del sentido personal y de la significación vital que tiene ese conocimiento, transformador de la realidad; y a la vez, de autotransformación. Engloba la personalidad como un todo, es integral, sistémico, donde se configuran unidades de sentido, siendo en esencia participativo, de interacción y colaboración.

La manera en cómo se asume la lógica expresada hasta el momento, conduce a la comprensión del sentido de la enseñanza y el aprendizaje junto al desarrollo personológico. Por lo tanto, demanda la búsqueda de acciones de intervención promoviendo la participación activa estudiantil y la integración consciente de su proceso de desarrollo integral en el proceso de enseñanza-aprendizaje.

Debido a lo anterior, un tema que ha centrado el interés de las personas investigadoras ha sido el relacionado con los enfoques de aprendizaje, estos han sido definidos por Pérez 
Cabaní (2000, p. 43) como: "la intención que orienta la actividad de los estudiantes en un proceso complejo, que incluye simultáneamente consistencia y variabilidad".

Se distinguen tres tipos de enfoques. El enfoque superficial les permite a la estudiante y al estudiante cumplir con la tarea con un mínimo de esfuerzo y reproduce la información. Estudia sin reflexionar, trata el contenido como partículas aisladas, memoriza los datos y procedimientos, encuentra dificultades para dar sentido a las nuevas ideas que se presentan, siente presión y agobio sin motivo aparente.

El segundo enfoque es el estratégico que concibe que la persona estudiante organiza la información para conseguir el mejor resultado posible con un esfuerzo constante en el estudio; buscando las condiciones materiales adecuadas, gestiona el tiempo y el esfuerzo, presta atención a las demandas de la evaluación.

El tercer enfoque es el profundo, considera que la persona transforma la información para relacionar las ideas con el conocimiento previo, busca modelos y principios implícitos, comprueba la coherencia del proceso, examina la lógica y los argumentos de manera cautelosa y crítica. Es activa y participativa.

A pesar del valor de estas ideas y la relación que guarda con el éxito en el proceso docente, se considera que su compromiso con el constructivismo sólo permite enfocar el resultado intelectual de la población estudiantil, perdiendo de vista otros elementos centrales en el aprendizaje como los relacionados con lo afectivo, lo volitivo y el carácter integral de la persona en su proceso de aprendizaje. En la sociedad actual: ¿Se demanda un aprender a aprender o un aprender a ser?

Gustavo Torroella (2002) aborda la presencia de un enfoque actual de educación para la vida o pedagogía del ser que propende al desarrollo integral de la personalidad, de las potencialidades de las personas y a la plenitud humana. Se caracteriza por los siguientes principios rectores: la educación centrada en estudiantes, en su atención y comprensión; el respeto, aceptación y amor al educando, como actitud fundamental del educador; la vitalización de la escuela, su vinculación con la vida en el doble sentido de abrir, de llevar la escuela a la vida y traer e introducir la vida en la escuela, la escuela como un taller para la Volumen 9, Número 2, Año 2009, ISSN 1409-4703 
vida, para la vida naciente y creadora frente al embalsamamiento y momificación del pasado en la escuela tradicional; la actividad frente a la pasividad, la inercia y el bostezo de la escuela tradicional, pero, principalmente, la auto-actividad, es decir, la generada y dirigida por la propia persona; la espontaneidad creadora, frente al formalismo, la rigidez y el autoritarismo, la libertad y la espontaneidad para tener iniciativas, para expresarse y elegir; finalmente, la educación para la vida está orientada hacia la comunidad, hacia la sociedad, para una convivencia armoniosa y cooperativa, para promover la responsabilidad y la conducta prosocial.

El primero de estos enfoques de aprendizaje reúne en sí aspectos como aprender a autocuidarse y promover la salud integral; aprender a conocerse a sí mismo y mejorar la autoestima, desarrollar la voluntad, aprender a orientarse, formar una jerarquía de valores o sentidos de la vida y elaborar proyectos de vida.

El segundo sistema está compuesto por un aprender a socializarse, a ser una persona social y miembra activa y participante creativa de la sociedad de la pequeña patria que es la comunidad en que se vive y de la grande que es el país. Sin olvidar que en última instancia Patria es Humanidad, aprender a expresarse y a comunicarse con las demás personas; aprender a convivir amistosa y cooperativamente (familiares, compañeras y compañeros, pareja, etc.). Forman parte de este sistema aprender a elegir pareja y establecer una unión sexual, matrimonial y familiar estable y satisfactoria, y aprender a mejorar las relaciones humanas y las comunicaciones interpersonales.

Un tercer sistema de aprendizaje engloba aprender a estimar, a disfrutar y a crear los valores positivos de la vida: belleza, amor, bondad, verdad, justicia, dignidad, felicidad, etc.; aprender a pensar, trabajar y crear; aprender a enfrentar positivamente las situaciones de la vida; aprender las conductas racionales y constructivas frente a los problemas; aprender a enfrentar, compensar, vencer y superar los problemas, las frustraciones, el estrés y los fracasos de la vida.

Los aprendizajes básicos no constituyen una suma de aprendizajes diversos, sino un sistema cuyo eje o columna vertebral es la orientación de valores o sentido de la vida del sujeto. 


\section{Radiografía Número 2. El Constructivismo v.s. el enfoque Histórico-Cultural}

Encuadradas dentro del enfoque psicológico constructivista, las estrategias de aprendizaje son elementos que permiten explicar la intención de docentes e investigadores en lograr el desarrollo intelectual estudiantil y el perfeccionamiento del sistema docente. Para su comprensión es necesario un análisis de la posición constructivista de la psicología.

El cognositivismo como enfoque psicológico, no pudo desentrañar la compleja naturaleza relacionada con los fenómenos del aprendizaje y el desarrollo, dando paso al constructivismo como una nueva forma de abordar esta realidad que, sorteando los intentos anteriores, propone una convergencia de varios enfoques para abordar este proceso.

En sus orígenes surge como una corriente epistemológica preocupada por discernir los problemas de la formación del conocimiento en el ser humano. Ante la pregunta ¿qué es el constructivismo? puede decirse que es la idea que mantiene que la persona tanto en los aspectos cognitivos y sociales como en los afectivos no es un mero producto del ambiente ni un simple resultado de sus disposiciones internas, sino una construcción propia que se va produciendo día a día como resultado de la interacción entre estos dos factores. En consecuencia, el conocimiento no es una copia fiel de la realidad, sino una construcción del ser humano. ¿Con qué instrumentos realiza la persona dicha construcción? Fundamentalmente con los esquemas que ya posee, es decir, con lo que ya construyó en su relación con el medio que le rodea.

Su núcleo teórico básico está sustentado en cuatro principios o categorías de análisis:

> Constructivismo: Categoría con poder de inclusión donde pueden integrarse diferentes corrientes psicológicas que ponen acento en la actividad del sujeto, en especial en las teorías de corte cognoscitivo. El aprendizaje debe iniciar con la actividad del alumno. Este tipo de actividad constructiva es mediatizada por las condiciones institucionales y tiene como finalidad última la construcción de significados.

> Aprendizaje significativo: Se considera que se ha logrado un aprendizaje cuando la estudiante y el estudiante le puede atribuir al contenido un significado y esto solamente lo consigue si se logran establecer relaciones sustantivas entre el nuevo material y el conocimiento previo. Para que el aprendizaje sea significativo debe Volumen 9, Número 2, Año 2009, ISSN 1409-4703 
cumplir dos condiciones: el contenido debe ser potencialmente significativo, tanto a nivel lógico como psicológico y el estudiante debe presentar una disposición favorable.

$>$ Esquemas de conocimientos: representación que manifiesta una persona en un momento determinado de su historia sobre una parcela de la realidad. Presenta carácter integrador y totalizador.

> Interactividad: se encuentra sustentado en los principios histórico-culturales de Zona de Desarrollo Próximo y la Ley Genética General del desarrollo cultural. Se convierte en un principio explicativo a través de la incorporación de otros elementos a los marcos de la actividad en el contexto educativo.

El postulado básico del constructivismo sitúa la clave del proceso enseñanzaaprendizaje en la interacción entre los tres elementos del triángulo interactivo: estudiante-docentecontenido. En este triángulo la actividad educativa docente es de ayuda en la construcción de significados. La opción básica que asume la concepción constructivista es la enseñanza adaptada, que propone hacer frente a la diversidad mediante la utilización de métodos de enseñanza diferentes en función de las características individuales de cada estudiante.

La diversidad humana se basa en la concepción interaccionista de las diferencias individuales. Desde esta perspectiva se reconocen la exigencia de características intrínsecas a la propia persona - determinadas posiblemente por su carga genética - y de reconocer, así mismo, el papel que juega el medio - con sus mediadores, familia, profesores, medios de comunicación- en las diferentes situaciones en que se encuentra la persona. Las diferencias individuales son el fruto de la interacción entre las características internas y las características del medio externo, por ello la diversidad humana solo se puede entender y tratar adecuadamente si se consideran ambos factores en interacción.

Algunas ideas de esta concepción son coincidentes con el planteamiento de la enseñanza adaptada, para muestra tenemos: conocimientos previos; conceptos como el de la ayuda contingente, plasticidad o adaptación de la intervención pedagógica a la actividad estudiantil; el grado de desarrollo o capacidad general de cada estudiante; la motivación para aprender, significativamente, así como sus intereses personales, y algunos más. 
Desde una concepción constructivista de la educación, y dentro del acto didáctico, es importante considerar los procesos de enseñar a pensar y de enseñar a aprender que, en definitiva, son mecanismos que favorecen el conocimiento de uno mismo y ayudan al aprendiz a identificarse y a diferenciarse de las demás personas. De este modo, las estudiantes y los estudiantes llegarían a ser conscientes de sus motivaciones e intenciones, de sus propias capacidades cognitivas y de las demandas de las faenas académicas, llegando a ser capaces de controlar sus recursos y de regular su posterior actuación.

Las limitaciones reconocidas por Kraftchenko, O. y Hernández, H. (2000), y que para nuestro criterio tienen incalculable validez, son:

> Subjetivismo: se considera el aprendizaje como el resultado de una construcción individual y no como el reflejo de la realidad objetiva.

$>$ Agnosticismo: el sujeto no llega a elaborar los conocimientos objetivos, cuya validez depende solo del hecho de que sean viables o producto de las interrelaciones.

$>$ Restricción cooperativa: subvaloración del papel de madres, padres y coetáneos en la construcción del conocimiento.

> Espontaneismo: se pone énfasis en la construcción de las estructuras y del conocimiento como proceso muy relacionado al de maduración.

> Privilegiar el método en relación con el contenido y otros criterios pedagógicos.

$>$ No diferencia en cuanto a la formación de conceptos científicos y no científicos, obviando el carácter intencional y dirigido del proceso de formación del concepto científico.

$>$ Concepción de un diseño curricular basado en intereses y posibilidades de las estudiantes y los estudiantes, subestimando áreas de conocimiento y habilidades que son importantes desarrollar.

> Relativismo en la educación moral conduce a la acentuación del individualismo en el marco de una supuesta colaboración.

El constructivismo es una convergencia de principios explicativos del proceso de enseñanza aprendizaje, totalmente abierta a matices diversos, ampliaciones y correcciones. El constructivismo mantiene que la actividad, física y mental, es en efecto lo que le permite desarrollarse progresivamente, sentir y conocerse a sí mismo y a la realidad externa. Ahora 
bien, este proceso de constructivismo progresivo, que tiene lugar como resultado de la actividad, no se basa en la nada, sino en el medio que envuelve a la persona.

Siguiendo las ideas de L. S. Vigotsky (1989) se aprecia que el constructivismo no integra la realidad al proceso de aprender, solo depende de la actividad mental del estudiante, la presencia de esquemas, necesitando, por tanto, un nivel determinado de maduración de las estructuras mentales. Se considera que sobrevalora el carácter intelectual de la subjetividad.

Tampoco existe una comprensión integral del proceso de desarrollo humano limitando sus intentos de comprender y estimular el crecimiento personal al dominio de algoritmos que permiten solucionar determinada situación problémica. Podría cuestionarse: ¿el desarrollo es un proceso de autoorganización cognitiva?

Existe una estrecha relación teórico-metodológica entre el constructivismo y las estrategias de aprendizaje. Nuestra opinión enfatiza en que se debe concebir el aprendizaje desarrollador desde un enfoque histórico-cultural, asumiendo los conceptos de desarrollo, enseñanza, cultura, significación, etc. Además, la forma de actuar tiene que ser congruente con lo antes expuesto, ya que al asumir el modelo vigostkiano, como enfoque epistemológico, nos lleva a un pensar-actuar en relación con lo teórico-metodológico en cuanto a este enfoque.

\section{Radiografía Número 3. Visión de las estrategias de aprendizaje}

El actual interés por el tema de las estrategias de aprendizaje es en parte promovido por las nuevas orientaciones psicopedagógicas que generan la Reformas Educativas en diversas latitudes. A lo largo de las décadas se han hecho aportaciones significativas desde diferentes concepciones y modelos que han matizado el actual estado sobre la cuestión.

El término estrategias ha estado asociado a las acciones bélicas, y recogido en textos griegos y latinos. Actualmente trasciende estos marcos y escuchamos hablar de estrategias políticas, económicas, financieras, etc., refiriéndose a un conjunto de acciones que debemos realizar para alcanzar un objetivo o meta. Al relacionarse con el aprendizaje surge entonces como "un conjunto de acciones que se realizan para obtener un objetivo de aprendizaje" Volumen 9, Número 2, Año 2009, ISSN 1409-4703 
(Monereo, 1999, p. 90). Monereo (1999) plantea que las estrategias de aprendizaje son procesos de toma de decisiones -conscientes o intencionales - en las cuales la población estudiantil elige y recupera de manera coordinada, los conocimientos que recesita para cumplir una determinada demanda u objetivo, dependiendo de las características de la situación educativa en que se produce la acción.

El concepto propuesto por C. Monereo (1999) es reformulado posteriormente al plantear que las estrategias de aprendizaje son procesos de toma de decisión, conscientes e intencionales, que consisten en seleccionar los conocimientos conceptuales, procedimentales y actitudinales necesarios para cumplir un determinado objetivo siempre en función de las condiciones de la situación educativa en que se produce la acción.

De manera general se afirma que los conceptos propuestos presentan elementos similares, como ser procedimiento o secuencia de acciones, por lo tanto, son actividades conscientes y voluntarias. Pueden incluir varias técnicas, operaciones o actividades específicas y persiguen un propósito determinado: el aprendizaje y la resolución de problemas académicos. Se realizan flexiblemente y son instrumentos con cuya ayuda se potencian las actividades de aprendizaje y solución de problemas. Se convierten en instrumentos socioculturales aprendidos en contextos de interacción con alguien que sabe más.

César Coll (1999) refiere que el conocimiento asociado a las estrategias de aprendizaje ocurre respecto a tres ideas fundamentales:

$>$ El estudiante como máximo responsable de su proceso de aprendizaje, ya que es este quien construye el conocimiento y nadie puede sustituirlo en esta tarea.

$>$ La actividad constructiva del estudiante se aplica a contenidos que ya posee en un grado de elaboración considerable, es decir, la práctica de los contenidos que constituyen el núcleo del aprendizaje escolar son conocimientos y formas culturales.

$>$ La función del docente es de facilitador del proceso de construcción del conocimiento.

A pesar de existir aproximaciones conceptuales, se halla una diversidad amplia en cuanto a las clasificaciones. Una de las propuestas que, consideramos, reúne mayor cantidad de estrategias de aprendizaje y, a la vez, es sumamente exhaustiva en cuanto a los criterios de 
diferenciación la ofrecen Díaz-Barriga y Hernández (2002), quienes realizan la clasificación de las estrategias de aprendizaje utilizando diferentes indicadores.

En la clasificación propuesta por Valle (2000) se resalta que las estrategias cognitivas son un conjunto de habilidades que se utilizan para aprender, codificar, comprender y recordar la información al servicio de una determinada meta de aprendizaje. Se distinguen tres clases de estrategias: estrategias de repetición, de elaboración y de organización. Las primeras consisten en pronunciar, nombrar o decir, de forma repetida, los estímulos presentados dentro de una tarea de aprendizaje. Mecanismo de memoria que activa los materiales de información para mantenerlos en la memoria a corto plazo. Las estrategias de elaboración integran los materiales informativos, relacionados con la nueva información, con la ya almacenada en la memoria. Las estrategias de organización intentan combinar los elementos informativos seleccionados de un modo coherente y significativo. Se pueden abordar otro tipo de estrategias cognitivas de selección, cuya función principal es la de escoger aquella información más relevante con la finalidad de facilitar su procesamiento.

Las estrategias metacognitivas requieren consciencia y conocimiento de variables de la persona, de la tarea y de la estrategia. La metacognición regula de dos formas el uso eficaz de las estrategias: en primer lugar, para que un individuo pueda poner en práctica una estrategia, antes debe tener un conocimiento de estrategia específica y saber cómo, cuándo y por qué debe usarla. En segundo lugar, su función autorreguladora hace posible observar la eficacia de las estrategias elegidas y cambiarlas según las demandas de la tarea.

Por último, las estrategias de manejo de recursos son estrategias de apoyo que incluyen diferentes tipos de recursos que contribuyen a la resolución de la tarea. Tienen como finalidad sensibilizar al estudiante con lo que va a aprender, integrando tres ámbitos: la motivación, las actitudes y el afecto. Estas estrategias incluyen el control del tiempo, la organización del ambiente de estudio y el control de los esfuerzos, entre otros.

Se concuerda con Straka (1997, p. 69) cuando plantea que las estrategias de aprendizaje: "describen al ser motivado y autodirigido que aprende como una persona que dispone del correspondiente saber fundamental y que está dispuesta a, y es capaz de, planificar su aprendizaje en forma autónoma y bajo su propia responsabilidad; de organizarlo". 
Este autor reconoce que las estrategias de aprendizaje se centran en tres categorías que son: la administración de recursos (organización de las condiciones básicas, personales e impersonales, del aprendizaje y el trabajo, obtención de información, estructuración del trabajo, colaboración con otras personas); determinación de la secuencia (planes orientados a los objetivos de un proceso de aprendizaje y trabajo, planificación del tiempo, planificación de las etapas y planificación de las fases de distensión); y la implementación (incluye la repetición, estructuración y elaboración de los contenidos formativos). No obstante nuestras reflexiones, cabría preguntarnos: ¿existe alguna forma puramente racional de enfrentar la vida?

Para otros autores como Monereo, C. (1999) y Coll, C. (1999) es importante la toma de decisión de estrategia que realiza el estudiantado cuando se enfrenta a la actividad. Dentro de los criterios que cada estudiante debe realizar debe proponerse una adecuada valoración en función de varios indicadores. Primero, los contenidos de aprendizaje (tipo y cantidad): la estrategia utilizada puede variar en función de lo que se tiene que aprender, (datos o hechos, conceptos, etc.), así como de la cantidad de información que debe ser aprendida. Los conocimientos previos que tenga sobre el contenido de aprendizaje: si el estudiante quiere relacionar distintos tipos de elementos que existen y clasificarlos, es necesario tener unos conocimientos más amplios que saber el nombre. Además, las condiciones de aprendizaje (tiempo disponible, la motivación, las ganas de estudiar, etc.) son criterios muy interesantes. En general, se puede decir que a menos tiempo y más motivación extrínseca para el aprendizaje, más fácil es usar estrategias que favorecen recordar, literalmente, la información (como el ensayo), y menos las estrategias que dan significado a la información o la reorganizan (estrategias de elaboración o de organización). Por último, el tipo de evaluación al que va a ser sometido: en la mayoría de los aprendizajes educativos la finalidad esencial es superar los exámenes; por tanto, será útil saber el tipo de examen al que se va a enfrentar.

El método más usual para estimular la enseñanza directa de las estrategias, es el modelamiento seguida de una práctica guiada. En el modelamiento se entiende que se va más allá de la imitación. Se trata de que el control y la dirección, que en un principio son ejercidos por docentes, sean asumidas por estudiantes. El medio utilizado para conseguir esto es la verbalización. 
Si se analiza esta propuesta surgen las siguientes interrogantes: ¿Dónde queda la individualidad de cada estudiante? ¿Se puede hablar de ayuda pedagógica o de imitación mecánica y reproductiva? ¿Cómo se interpreta el papel activo de cada estudiante en el proceso enseñanzaaprendizaje?

Cuando nos enfrentamos a determinada estrategia de aprendizaje de cualquier tipo, esta se nos presenta como la posibilidad de resolver nuestro problema en relación con los contenidos y habilidades que tenemos que interiorizar. Se convierte en un listado de consejos generales supuestamente útiles para cualquier contexto de aprendizaje. Pero si reflexionamos con lo que está ocurriendo a nuestro alrededor pueden surgir otras tantas preguntas como las siguientes:

¿Para qué tipología de problema es aplicable la técnica?

¿Qué relación epistemológica guarda con los contenidos disciplinares que maneja?

¿Qué conocimientos conceptuales y procedimentales y qué esfuerzo cognitivo se requiere para su aplicación?

¿En qué aspectos resulta más adecuada su aplicación que otras técnicas y en cuáles no?

¿Respecto a qué variables deben tomarse las decisiones y qué tipo de dificultad puede anticiparse?

¿Qué variaciones o alternativas de uso permite?

¿Qué tipo o calidad de aprendizaje producen? ¿Bajo qué condiciones?

¿Qué peligros entraña?

¿Se promueve el desarrollo personológico mediante la utilización de estrategias de aprendizaje?

\section{Valoraciones de interés}

El surgimiento de las estrategias de aprendizaje sirvió para dar respuesta a la necesidad de optimizar los procesos mentales en la actividad de enseñanza-aprendizaje en la escuela, pautar modelos para aprender, lograr resultados de aprendizaje en cuanto a conocimientos con el mínimo de esfuerzo. Su utilización es un paso de avance ante la inconsistencia del modelo reactivo y es un esfuerzo por encontrar a cada estudiante en su proceso de aprendizaje, que anteriormente no había sido tomado en cuenta. 
Durante una década de utilización necesaria por la mayoría de los centros de enseñanza, sobre todo del mundo desarrollado, consideramos necesario abordar las limitaciones que a nuestro entender estas presentan y las hacen inconsistentes en la búsqueda del desarrollo integral del sujeto.

Las estrategias de aprendizaje están estrechamente relacionadas con la idea de que el lugar por excelencia para aprender es la escuela, sin considerar otros contextos de enseñanza y formas autónomas de aprendizaje. Se considera que los contextos de aprendizaje en los que se desenvuelve el sujeto son múltiples y debe poseer más que algoritmos de enfrentamiento, potencialidades integradoras de desenvolvimiento y estructuración de sentidos y construcción de significados, integrando aspectos afectivos y cognitivos.

A pesar de ser un pensamiento que ha evolucionado en relación con el papel que debe tener la estudiante y el estudiante en su proceso de enseñanza aprendizaje, han sido estas mismas estrategias las que siguen elevando el papel activo del sujeto, conocedor de sí mismo, examinando que es insuficiente reflexionar solo sobre procedimientos o instrumentos aplicados en una situación o sobre los recursos cognitivos por emplear, sino partir de la autorregulación a la búsqueda de los elementos dinámicos, afectivos motivacionales, volitivos, comportamentales que se configuran en la historia individual del sujeto. Entendemos que el reconocimiento del carácter activo, al y como ha sido tratado, queda limitado, ya que tanto en el dominio del proceso de apropiación de estrategias de aprendizaje, como en su utilización es determinante el papel docente y solo en un segundo momento es que cada estudiante asume de manera gradual el control del proceso.

De igual manera queda limitado el papel de los sentidos psicológicos, lo cual permitió ubicar la personalidad de la persona aprendiz como centro del proceso de aprendizaje, desde los contenidos, posibilitando la búsqueda por parte del sujeto de sus propios epistemas, la construcción de sus significados, etc.

Este tipo de estrategias no estimula el trabajo con las interacciones alumno-alumno, otorgándole poco valor a las mismas, aunque en algún caso solo reconoce su presencia. Siendo consecuentes con el concepto de Zona de Desarrollo Próximo de L. S. Vigotsky consideramos que en el aprendizaje la influencia de cualquier persona significativa, en la Volumen 9, Número 2, Año 2009, ISSN 1409-4703 
solución de una tarea, posibilita el posterior dominio de esos instrumentos psicológicos y la capacidad de que el estudiante pueda realizar la tarea.

Para lograr una apropiación desde lo personológico en el uso de las estrategias de aprendizaje debe lograrse una relación visible con las expectativas del sujeto, facilitando que el aprendiz integre su estructuración futura de la personalidad. De esta forma el sujeto se compromete con el éxito en sus proyecciones. El sujeto sentirá como necesario su uso no solo en su vida escolar, sino en la cotidianeidad, permitiendo que experimente satisfacción, autoconfianza, búsqueda y se autorregule en función de ello.

Las estrategias de aprendizaje son asimiladas como saberes y no pueden ser utilizadas en situaciones distintas, como tampoco parten de un diagnóstico del sujeto psicológico que las utilizará, para ello, se propone para un tipo de habilidad o problema para todos los sujetos. Cada sujeto debe partir de su conocimiento y la amplitud para la cual se ha diseñado la estrategia, utilizándola en la vida cotidiana, al enfrentar disímiles condiciones.

\section{A modo de epílogo}

Partiendo de las limitaciones que se han señalado en los modelos de estrategias de aprendizaje, tanto teórica como metodológicamente, se propone el análisis de una nueva forma de concebir las estrategias de aprendizaje. Esta visión es el resultado de una valoración consecuente y reflexiva de los enfoques teóricos y su puesta en práctica, así como el acercamiento consciente al modelo materialista dialéctico del desarrollo humano, coincidente con el enfoque histórico-cultural de la psicología. Se han tomado en cuenta las características de la sociedad del conocimiento actual, fiel reflejo de un mundo complejo, dinámico y en constante desarrollo. Se considera que no es una epistemología acabada, aunque cuenta con exigencias que orientan a la estimulación del desarrollo personológico desde la dirección del proceso enseñanza-aprendizaje.

Se conciben estas Estrategias de Aprendizaje de nuevo tipo como un proceso complejo, donde los contenidos psicológicos se combinan de manera peculiar, a partir del carácter activo que asume la población estudiantil_en su proceso de aprendizaje, logrando niveles superiores de autorregulación del comportamiento. Su esencia radica en la posibilidad de Volumen 9, Número 2, Año 2009, ISSN 1409-4703 
autoestimulación de los mecanismos y configuraciones psicológicas superiores expresadas en la reflexión, motivación y el autodesarrollo de los estudiantes.

Considerar a las Estrategias de Aprendizaje como una nueva modalidad de estrategias de aprendizaje constructivista es un error, pues existe una ruptura radical entre ellas. Las estrategias de aprendizaje son conceptualizadas como procesos de toma de decisiones para la selección de conocimientos, habilidades, herramientas, con objetivos centrados en el proceso docente. Las Estrategias de Aprendizaje trascienden esta idea al ser concebidas como una vía para promover la formación y estructuración de los contenidos psicológicos altamente elaborados por estudiantes, de manera tal que permite la autoestimulación de mecanismos y formaciones psíquicas nuevas. En el grupo estudiantil se logra estimular la autonomía en su proceso de desarrollo. Así, emergen dos características de las Estrategias de Aprendizaje: por un lado, la autoestimulación del desarrollo integral de las personas estudiantes y, por otro, la autodeterminación a lo largo del proceso de aprendizaje y desarrollo, utilizándolos para lograr los objetivos/metas personales y profesionales, recursos cognitivos, afectivos, volitivos.

La población estudiantil asume un papel determinante en el uso de estrategias desarrolladoras. Su rol parte de la implicación en situaciones que cada vez exigen niveles superiores de estructuración psicológica, con despliegues desarrolladores de mayor envergadura. Valora su desenvolvimiento en la misma, existe un elevado compromiso con el desarrollo personal y profesional.

En las Estrategias de Aprendizaje se refuerza la idea de ser un mecanismo dinámico causal de dominio de la conducta y potenciador del desarrollo personológico. El diseño dinámico se centrará en los recursos, en la calidad de los componentes de significados, sentidos e interactivos del proceso, considerando al sujeto como agente activo a nivel metacognitivo, motivacional y autorregulador del comportamiento.

Se hace referencia a una intencionalidad autorregulada en la que involucran las metas, expectativas, decisiones, esfuerzos que, mayormente, se implican como sistema complejo e integrado cuando se presenta en situaciones de aprendizaje. 
La reflexión expresada en el uso de Estrategias de Aprendizaje permite la implicación del sujeto en su dinámica psicológica a la hora de enfrentar diversas situaciones. Como sujeto psicológico las formas de realizar la actividad, buscar soluciones, valorar, crear, planificar y trascender su nivel actual de desarrollo parten de un acercamiento a la dinámica configuracional de su personalidad antes de buscar elementos externos para solucionar la situación, permitiéndole contar con recursos que conforman contenidos psicológicos altamente configurados, donde adquieren un rol significativo procesos de características diferentes a los intelectuales. Las herramientas psicológicas juegan un papel determinante en esta nueva estructuración y regulación del comportamiento.

Desde un enfoque desarrollador, auando se aprende se posibilita la reflexión del sujeto psicológico que se implica en la situación provocando no solo una valoración de la situación o los recursos intelectuales propios, sino que reflexiona y enriquece contenidos y funciones psíquicas superiores en su configuración reguladora del comportamiento.

La reflexión sobre sí mismo, idea básica del proceso de desarrollo, enriquece formaciones psíquicas superiores como el autoconcepto y la autovaloración, implicando definiciones en la estructuración reguladora del comportamiento. El sujeto psicológico en la reflexión actúa desde lo personológico, lo integral y lo desarrollador.

Autores como F. González han enfatizado en la reflexión como una cualidad del pensamiento teórico que se revela, sobre todo, en la solución de problemas, nosotros creemos que estos supuestos no contradicen su valoración personológica, cuando la estudiante o el estudiante, en la elaboración de sus motivaciones, transforma cognitivamente las necesidades que representan su vinculación consigo mismo y con el mundo. Como expresa Labarrere A. una de las particularidades más sobresalientes del comportamiento inteligente radica en su actividad cognoscitiva autocontrolada o autorregulada.

La motivación juega un papel determinante dentro de las Estrategias de Aprendizaje, ya que este elemento caracteriza la integridad y disposición positiva la persona ante el proceso de aprendizaje. Este elemento, además de ser importante en el proceso personalizado, logra nuevas formas de autorregulación del comportamiento, configuración jerárquica, riqueza de contenido y sentido psicológico, entre otras. Considerado como uno de los mecanismos Volumen 9, Número 2, Año 2009, ISSN 1409-4703 
psicológicos que son ejes de la propuesta desarrolladora, la motivación encauza el esfuerzo del estudiante y pauta la dinámica psicológica al incluir en su jerarquía, necesidades, motivos, intereses, ideales, conflictos y otros procesos psicológicos que de manera personalizada regulan su comportamiento.

La motivación del sujeto implicado en estrategias desarrolladoras gana en riqueza psicológica, traducida en formaciones de sentidos personalizados, aumento de la motivación intrínseca y el conocimiento sobre sí y el contexto, presencia de elementos aportadores a las intenciones profesionales, entre otras. La motivación ha ganado en fuerza movilizadora de otros recursos, logra implicar al sujeto en nuevos retos y situaciones y volver sobre su proceso de desarrollo, por lo que se puede hablar de una dinámica de cambio en cuanto a la motivación del sujeto en su proceso de aprendizaje autodesarrollador, de una motivación personalizada a una automotivación, convertida en la búsqueda de sentidos enriquecidos, de la jerarquía motivacional presente en el estudiante. Los motivos intrínsecos son inherentes a la propia esencia de la actividad y satisfacen necesidades del sujeto vinculadas directamente con la misma.

Los elementos motivacionales se convierten en expresión activa de elaboración intelectual, son portadores de una elevada carga emocional, que, evidencia su potencial motivacional sobre el comportamiento, estando orientadas a la regulación del comportamiento, teniendo su expresión en esta dirección en objetivos, valoraciones y otras formas activas de autodeterminación.

El autodesarrollo es considerado como un mecanismo psicológico con gran impacto en la estimulación del desarrollo desde estas estrategias de aprendizaje. A lo largo del proceso, el sujeto se conoce mejor, reflexiona sobre sí, se motiva e implica orientado por su carácter prospectivo, se provoca el paso de una estimulación externa del sujeto, ya sea por otro sujeto o él mismo apoyado en elementos del contexto, a una autoestimulación del desarrollo, con mecanismos autoeducativos y de autoperfeccionamiento. Se considera que los estudiantes pueden lograr una dinámica que oriente el desarrollo al crecimiento autopotenciado. 
Cuando la persona actúa desde estrategias desarrolladoras constantemente valora, no solo el resultado de la actividad, sino la implicación en el proceso, sus logros y deficiencias. Se han incentivado la crítica y la autocrítica, el autoperfeccionamiento, y otros mecanismos psicológicos que le permiten inmediatamente superar las limitaciones, creando estados de inconformidad consigo mismo. Estas valoraciones realizadas se configuran de manera armónica en la regulación del comportamiento estudiantil a lo largo de su proceso de aprendizaje, lográndose activar elementos de su dinámica desarrolladora.

En el proceso de aprendizaje desarrollador se logra que confluyan elementos organizados en los contenidos psicológicos. Los contenidos personalizados y configurados en las representaciones sobre la profesión, en el conocimiento previo, las vivencias, entre otras, con los contenidos psicológicos estructurados en funciones superiores como las intenciones profesionales, ideales, sentido de la vida, etc., son reestructuradas de manera activa por la persona en su proceso de aprendizaje, adquiriendo la esencia ontogenética del sujeto.

Esta relación nutre las situaciones de aprendizaje con nuevas configuraciones, en las cuales el sujeto se hace consciente, se reestructura, y en función de ello regula su comportamiento. Se hacen latentes contenidos y configuraciones que permiten actualizar las representaciones y sentidos psicológicos actuantes en el sujeto. Las Estrategias de Aprendizaje para el Desarrollo permiten la movilización de recursos personológicos, haciendo conscientes la estructuración futura partiendo del análisis de los niveles alcanzados en su desarrollo psicológico.

Las Estrategias de Aprendizaje para el Desarrollo son una herramienta para el proceso de autoeducación de la personalidad. Autoeducar, en tanto que el sujeto se proponga, realice y valore un sistema de acciones encaminadas a autoestimular su desarrollo integral de manera autónoma, trascendiendo el marco docente con intenciones de desarrollo. Las estrategias desarrolladoras se convierten en ese sistema de acciones de carácter procesual, donde en cada tarea, el estudiante forma y reestructura contenidos psicológicos que adquieren nuevos matices en la autorregulación del comportamiento y que difieren de las anteriores por dos elementos: su contenido y el papel desempeñado por el sujeto. 
Al abordar las Estrategias de Aprendizaje para el Desarrollo haciendo referencia a la regulación intencionada a lo largo del proceso de aprendizaje, donde cada estudiante asume el papel de autoestimulador de su desarrollo personológico y su aprendizaje autónomo. En esta regulación consciente los objetivos/metas guían el proceso y proponen el equilibrio entre los intereses personales y sociales como vía para lograr la autorrealización.

En el proceso de aprendizaje se activan recursos de naturaleza psicológica diferente y ante cada tarea docente se implica de manera integral el sujeto que aprende más allá de su propia tarea docente, con su desarrollo personológico. Las Estrategias de Aprendizaje para el Desarrollo permiten una construcción de significados y formación de sentido personal que enriquece los contenidos psicológicos y su dinámica estructural y funcional.

Al ser utilizadas por la población estudiantil, las Estrategias de Aprendizaje para el Desarrollo crean la necesidad de autoayuda, es decir, se convierten en el uso eficiente de instrumentos psicológicos que permiten la autorregulación del comportamiento y la autoestimulación del desarrollo.

La Universidad del siglo XXI debe de preparar a la persona para su vida futura, dotándolo de recursos autónomos, haciendo cuestionadora y gestora, abierta a las transformaciones, creativa, reflexiva y motivada. Nunca el mundo ha necesitado más de seres flexibles y trasformadores capaces de desentrañar los complejos procesos de la ciencia y la tecnología para ponerlos al servicio de la Humanidad. 


\section{Referencias}

Bozhovich, Ludmila. (1985). La personalidad y su formación en la edad infantil. La Habana: Pueblo y Educación.

Coll, César. (1999). Psicología de la instrucción: la enseñanza y el aprendizaje en la educación secundaria. Barcelona: Horsori.

Díaz-Barriga, F. y Hernández, G. (2002). Estrategias docentes para el aprendizaje significativo, una interpretación constructivista. México D.F.: Mc-GrawHill.

Kraftchenko, Otmara. y Hernández, Hilda. (2000). Constructivismo en tres direcciones. Vigotski: ¿constructivista? En Revista Cubana de Educación Superior. Vol. XX (3), 12-18.

Monereo, Charles. (1999). Concepciones sobre el concepto de estrategias de aprendizaje y sobre su enseñanza. El aprendizaje estratégico. (pp. 79-111). Madrid: Aula XXI. Santillana.

Pérez Cabaní, María Luisa. (2000). El aprendizaje escolar desde el punto de vista del alumno: los enfoques del aprendizaje. Psicología de la educación escolar. Madrid: Alianza.

Straka, Cerald. (1997). Un modelo de aprendizaje motivado y autodirigido. En Revista Educación. 55 (2), 20-33.

Torroella, Gustavo. (2002). La educación para la vida y el desarrollo humano, un reto para la escuela de hoy. Presentado en Taller del CEPES, 2002. Material fotocopiado.

UNESCO. (1998). Política y estrategias para la universidad latinoamericana del futuro. Material Fotocopiado.

Valle, Alberto. (2000). Las estrategias de aprendizaje utilizadas en el aula. Recuperado el16 de febrero del 2004, de http://www.monografias.com/trabajos5/teap/teap.shtml.

Vigotsky, Lev Semionovich. (1968) Pensamiento y Lenguaje. La Habana: Revolucionaria.

Vigotsky, Lev Semionovich. (1987). Historia del desarrollo de las funciones psíquicas superiores. La Habana: Científico-Técnica.

Vigotsky, Lev Semionovich. (1989). Obras Completas, Tomo V. Fundamentos de defectología. La Habana: Pueblo y Educación. 